\title{
SOLUTION OF A GENERALIZED SCHROEDER EQUATION IN TWO VARIABLES
}

\author{
C. F. SCHUBERT ${ }^{1}$ \\ (received 5 May 1964)
}

\section{Introduction}

If $f(z)$ is analytic at the origin, $f(0)=0$, and $f^{\prime}(0)=\lambda$, where $0<|\lambda|<1$, then Koenigs' [3] solution of Schroeder's equation $w(f(z))=$ $\lambda w(z)$, with multiplier $\lambda$, is given by $w(z)=\lim _{n \rightarrow \infty} \lambda^{-n} f^{n}(z)$. Here $f^{n}(z)$ denotes the $n$th iterate of $f(z)$, defined inductively as $f^{0}(z)=z, f^{n}(z)=$ $f\left(f^{n-1}(z)\right), n=1,2,3, \cdots$. More generally the solution $w(z)$ of Schroeder's equation is uniquely determined to within a multiplicative constant by the requirement that it be analytic at the origin. From the uniqueness it follows that if $g(z)$ is analytic at the origin, vanishes there, and commutes with $f(z)$, i.e., $f(g(z))=g(f(z))$, then $w(g(z))=\mu w(z)$, for some multiplier $\mu$. Since $w^{\prime}(0)=1$ for Koenig's' solution, it has an inverse locally, and we find that $g(z)$ is uniquely determined by its linear part; in fact $g(z)=w^{-1}(\mu w(z))$. In particular the integral iterates of $f$ can be put in the form $w^{-1}\left(\lambda^{n} w(z)\right)$ for integral $n$. Thus for any $\alpha$, real or complex, we may define $f^{\alpha}(z)$, consistent with the above definition when $\alpha$ is a positive integer, as $w^{-1}\left(\lambda^{\alpha} w(z)\right)$. In this manner any function $g(z)$ of the above type can be considered as an iterate of $f(z)$. Also if $\alpha, \beta, \neq 0$ are any two distinct points sufficiently close to the origin there exists an analytic function $g(z)$ which commutes with $f(z)$ such that $g(0)=0, g(\alpha)=\beta$. In fact $g(z)=w^{-1}\left(\chi^{w}(z)\right)$, where the multiplier $\chi=w(\beta)(w(\alpha))^{-1}$. These facts are all well known, e.g. [2] [5], and we shall establish analogous results in a more general situation.

If $|\lambda|=0,1$, or $f$ is a non-analytic transformation, the above discussion is no longer immediately applicable. For an excellent account in these cases see [8]. Particularly in the former case, the solution of Abel's equation $\varphi(f(z))=\varphi(z)+\alpha$ serves much the same purpose as $w(z)$. In the case of an analytic transformation of a space of two real or complex variables into itself, we shall show that even when Schroeder's equation has no analytic solution, the same nice behaviour evidenced above when $0<|\lambda|<1$, may still prevail. This will be done by applying an algorithm similar to Koenigs', to a

1 The research and preparation of this paper were supported (in part) by the National Science Foundation Grant GP-1988. 
functional equation which is an appropriate generalization of those of Schroeder and Abel. Specifically let $f(x, y)=\left(f_{1}, f_{2}\right)$ where

$$
\begin{aligned}
& x \rightarrow f_{1}(x, y)=a_{11} x+a_{12} y+\cdots \\
& y \rightarrow f_{2}(x, y)=a_{21} x+a_{22} y+\cdots
\end{aligned}
$$

be an analytic transformation of two dimensional complex space into itself. Here and subsequentiy a series of dots indicates a power series which converges absolutely in some neighborhood of the origin and contains only powers of order two and higher in the variables $x, y$, If the eigenvalues $\lambda_{1}, \lambda_{2}$, of the matrix $\left[a_{i j}\right]$ of coefficients of the linear part of $f$ are distinct, then a preliminary linear transformation reduces $f$ to the simpler form

$$
\begin{aligned}
& f_{1}(x, y)=\lambda_{1} x+\cdots \\
& f_{2}(x, y)=\lambda_{2} y+\cdots
\end{aligned}
$$

$$
0<\left|\lambda_{1}\right|,\left|\lambda_{2}\right|<1 \text {, and } \lambda_{1}^{p} \neq \lambda_{2}, \lambda_{2}^{q} \neq \lambda_{1},
$$

for all positive integers $p, q$ then Schroeder's equation

$$
\begin{aligned}
& w_{1}\left(f_{1}, f_{2}\right)=\lambda_{1} w_{1}(x, y) \\
& w_{2}\left(f_{1}, f_{2}\right)=\lambda_{2} w_{2}(x, y),
\end{aligned}
$$

has a formal power series solution, but the obvious generalization of Koenigs' solution does not exist unless $\left|\lambda_{1}\right|^{2}<\left|\lambda_{2}\right| \leqq\left|\lambda_{1}\right|$. By suitably interpreting Koenigs' algorithm, and other techniques, many authors $[1],[6],[7]$, have shown that if the conditions (2) are satisfied then (3) possesses a unique solution $w=\left(w_{1}, w_{2}\right)$ of the form $w_{1}=x+\cdots, w_{2}=y+\cdots$, i.e. in this case $f$ can be linearized by means of the transformation $w$. If $\lambda_{1}^{p}=\lambda_{2}$, for some positive integer $p$, then equating coefficients in (3) we find that it has no solution $w$ even as formal power series, Bellman's result [1]. Leau [4], has, however, shown in $n$ dimensions the existence of a solution having at best singularities of logarithmic type distributed on surfaces passing through the origin. The exact nature of his solution is not clear since it involves an arbitrary solution of Abel's equation.

We consider now the exceptional cases. If $\lambda_{1}=\lambda_{2}$, then in general $f$ can only be reduced to the form

$$
\begin{aligned}
& f_{1}(x, y)=\lambda x+y+\cdots \\
& f_{2}(x, y)=\lambda y+\cdots
\end{aligned}
$$

by a preliminary linear transformation. If $\lambda_{1}=\lambda^{D}, \lambda_{2}=\lambda,|\lambda| \neq 0,1$, then the form (1) can by a further transformation be reduced to

$$
\begin{aligned}
& f_{1}(x, y)=\lambda^{p} x+a y^{p}+x(\cdots)+y^{p}(\cdots), \\
& f_{2}(x, y)=\lambda y+\cdots,
\end{aligned}
$$


where the terms in brackets vanish at $(0,0)$. To see this, suppose $f_{1}$ in (1) has the form $f_{1}(x, y)=\lambda^{p} x+a_{2} y^{2}+\cdots+a_{p} y^{p}+\cdots$, where the terms represented by the second series of dots have no pure $y^{2}, \cdots, y^{p}$ terms. Let $t$ be the transformation $t(x, y)=\left(x+b_{2} y^{2}+\cdots+b_{p-1} y^{p-1}, y\right)$ and $t^{-1}$ its inverse. By direct substitution we find that for $k<p$ the coefficient of $y^{k}$ in the composite transformation $\left(t \circ f \circ t^{-1}\right)$, is of the form $\left(\lambda^{k}-\lambda^{D}\right) b_{k}+a_{k}+\varphi\left(b^{1}, \cdots\right.$, $\left.b_{k-1}\right)$ for $k>2$, and $\left(\lambda^{2}-\lambda^{p}\right) b_{2}+a_{2}$, for $k=2$, where $\varphi$ is a polynomial in the indicated $b$ 's with coefficients which are polynomials in those of $\boldsymbol{f}$. Solving successively for $b_{2}, \cdots, b_{p-1}$, we can determine the required transformation $t$. Our problem now is to characterize all analytic transformations $g$, with $g(0,0)=(0,0)$, which commute with $f$, and incidentally, define a set of analytic iterates $\left\{\boldsymbol{f}^{\alpha}\right\}$ of $\boldsymbol{f}$ for arbitrary $\alpha$ consistent with the integral iterates, defined of course as

$$
\begin{aligned}
& f^{0}(x, y)=(x, y) \\
& f^{n}(x, y)=f\left(f_{1}^{n-1}, f_{2}^{n-1}\right) \quad n=1,2,3, \cdots .
\end{aligned}
$$

If $f$ has the form (4) our method is to find an analytic solution $w=$ $\left(w_{1}, w_{2}\right)$ of the functional equations

$$
\begin{aligned}
& w_{1}\left(f_{1}, f_{2}\right)=\lambda w_{1}(x, y)+w_{2}(x, y) \\
& w_{2}\left(f_{1}, f_{2}\right)=\lambda w_{2}(x, y),
\end{aligned}
$$

using an algorithm analogous to Koenigs'. When $f$ has the form (5) we replace (6) by

$$
\begin{aligned}
& w_{1}\left(f_{1}, f_{2}\right)=\lambda w_{1}(x, y)+a\left(w_{2}(x, y)\right)^{p} \\
& w_{2}\left(f_{1}, f_{2}\right)=\lambda w_{2}(x, y) .
\end{aligned}
$$

Since our techniques in both cases are very similar we shall present the details for (6) and sketch them only for (7). We shall always assume that in (4) and (5) $0<|\lambda|<1$.

For any $\alpha$, real or complex, and some determination of $\lambda^{\alpha}$ we define the fractional iterate $f^{\alpha}$ of $f$ by the equations

$$
\begin{aligned}
& w_{1}\left(f_{1}^{\alpha}, f_{2}^{\alpha}\right)=\lambda^{\alpha} w_{1}(x, y)+\alpha \lambda^{\alpha-1} w_{2}(x, y) \\
& w_{2}\left(f_{1}^{\alpha}, f_{2}^{\alpha}\right)=\lambda^{\alpha} w_{2}(x, y),
\end{aligned}
$$

if $f$ has the form (4), and

$$
\begin{aligned}
& w_{1}\left(f_{1}^{\alpha}, f_{2}^{\alpha}\right)=\lambda^{\alpha p_{w}} w_{1}(x, y)+a \alpha \lambda^{\alpha p-p} w_{2}(x, y) \\
& w_{2}\left(f_{1}^{\alpha}, f_{2}^{\alpha}\right)=\lambda^{\alpha} w_{2}(x, y)
\end{aligned}
$$

if $f$ has the form (5). The $f^{\alpha}$ so defined will be shown to have the same properties as the integral iterates in a neighborhood of the origin and to be identical to them for integral $\alpha$. Further it will follow from the proofs, that $f^{\alpha}$ is analytic in $\alpha$ in any simply connected region of the $\alpha$ plane, omitting the origin, on which it is defined. 
In order to construct his solutions of Schroeder's equation, Leau [4], used functional equations similar to (6), (7), and proved the existence of analytic solutions by the method of dominant series. He did not however use his solutions to construct a family of fractional iterates, or to examine the more general question of classifying all analytic transformations which commute with $\boldsymbol{f}$.

\section{The case $\lambda_{1}=\lambda_{2}$}

The transformation $f$ now has the form (4). Without loss of generality for our purposes, we may assume that the power series representing $f$ converge absolutely for $|x|+|y| \leqq 1$. Choose a constant $k>\frac{1}{2}$ so that the sum of the absolute values of terms represented by dots in (4) are bounded above by $k(|x|+|y|)^{2}$, for $|x|+|y| \leqq 1$. We then have

Lemma 2.1. Let $s$ be real, $s^{2}<|\lambda|<s<1$, and put $\beta=1+(s-|\lambda|)^{-1}$. The region $\Omega$ defined by

$$
\Omega=\left\{(x, y) ;|x|+\beta|y|<(s-|\lambda|)[k(1+\beta)]^{-1}\right\},
$$

is then contained in $|x|+|y| \leqq 1$, is mapped into itself by $f$, and for all $(x, y) \in \Omega$, and positive integers $n$,

$$
\left|f_{1}^{n}\right|+\beta\left|f_{2}^{n}\right| \leqq s^{n}(1+\beta) .
$$

Proof. We have $(s-|\lambda|)<1<k(1+\beta)$, i.e., $(s-|\lambda|)[k(1+\beta)]^{-1}<1$, and $(s-|\lambda|)[\beta k(1+\beta)]^{-1}<1$, proving the first assertion. For $(x, y) \in \Omega$,

$$
\begin{aligned}
\left|f_{1}\right|+\beta\left|f_{2}\right| & \leqq|\lambda|(|x|+\beta|y|)+|y|+k(1+\beta)(|x|+|y|)^{2} \\
& \leqq|x|(|\lambda|+k(1+\beta)(|x|+|y|))+|y|(1+\beta|\lambda|+(1+\beta) k(|x|+|y|)) \\
& \leqq s(|x|+\beta|y|),
\end{aligned}
$$

since $(|x|+|y|)[k(1+\beta)] \leqq s-|\lambda|=\beta(s-|\lambda|)-1$. This proves the second assertion. The last follows by iterating this inequality for $(x, y) \in \Omega$.

The first result of this section is given by

TheOREM 2.1. Put

$$
w_{1}^{n}(x, y)=\lambda^{-n}\left[f_{1}^{n}(x, y)-n f_{2}^{n-1}(x, y)\right], w_{2}^{n}(x, y)=\lambda^{-n} f_{2}^{n}(x, y),
$$

for $n=1,2, \cdots$. Then $w=\left(w_{1}, w_{2}\right)=\lim _{n \rightarrow \infty}\left(w_{1}^{n}, w_{2}^{n}\right)$, exists and is an analytic solution of $(6)$ for $(x, y) \in \Omega$. w satisfies $w(0,0)=(0,0), w_{1}(x, y)=$ $x+\cdots, w_{2}(x, y)=y+\cdots$, and any other solution of (6) analytic at the origin with $w^{\prime}(0,0)=(0,0)$ is of the form $w^{\prime}=\left(\alpha w w_{1}+\beta w w_{2}, \alpha w w_{2}\right)$, for some constants $\alpha, \beta$. 
PrOOF. Provided the series on the right converges

$$
w_{2}(x, y)-y=\left(w_{2}^{1}-y\right)+\left(w_{2}^{2}-w_{2}^{1}\right)+\cdots .
$$

But for $n \geqq 0$ and $(x, y) \in \Omega$,

$$
\begin{aligned}
\left|w_{2}^{n+1}-w_{2}^{n}\right| & =|\lambda|^{-n-1}\left|f_{2}^{n+1}-\lambda f_{2}^{n}\right| \\
& \leqq|\lambda|^{-n-1} k\left(\left|f_{1}^{n}\right|+\left|f_{2}^{n}\right|\right)^{2} \\
& \leqq k(1+\beta)^{-1} \lambda^{-1}\left(s^{2} \lambda^{-1}\right)^{n}=b_{n}
\end{aligned}
$$

by the estimate of lemma 2,1 . The choice of $s$, ensures that $b_{n}$ is the $n$th term of a convergent series, and so $w_{2}$ certainly exists and is analytic in $\Omega$. Similarly for $(x, y) \in \Omega$, and $n>0$,

$$
\begin{aligned}
\left|w_{1}^{n+1}-w w_{1}^{n}\right| & \leqq|\lambda|^{-n-1}\left|\left(f_{1}^{n+1}-\lambda f_{1}^{n}-f_{2}^{n}\right)-n\left(f_{2}^{n}-\lambda f_{2}^{n-1}\right)\right| \\
& \leqq k|\lambda|^{-n-1}(1+\beta)^{2}\left[s^{2 n}+n s^{2 n-2}\right] .
\end{aligned}
$$

The right hand side of this inequality is the $n$th term of a convergent series, and repeating the above argument $w_{1}$ exists and has the stated properties. Also

$$
w_{1}^{n}\left(t_{1}, t_{2}\right)=\lambda w_{1}^{n+1}(x, y)+w_{2}^{n}(x, y) .
$$

Thus $w_{1}\left(f_{1}, f_{2}\right)=\lambda w_{1}(x, y)+w_{2}(x, y)$, and similarly $w_{2}\left(f_{1}, f_{2}\right)=\lambda w_{2}(x, y)$, as required.

To prove the last assertion, let $\boldsymbol{w}^{\prime}=\left(w_{1}^{\prime}, w_{2}^{\prime}\right)$ be another solution of (6) analytic at the origin. The transformation $w$ is invertible in a neighborhood of the origin and the inverse $w^{-1}=\left(w_{1}^{-1}, w_{2}^{-1}\right)$ satisfies

$$
\begin{aligned}
& \left(f \circ w^{-1}\right)_{1}(x, y)=w_{1}^{-1}(\lambda x+y, \lambda y) \\
& \left(f \circ w^{-1}\right)_{2}(x, y)=w_{2}^{-1}(\lambda x+y, \lambda y) .
\end{aligned}
$$

The composite transformation $\boldsymbol{w}^{\prime} \circ \boldsymbol{w}^{-1}$ thus satisfies

and similarly

$$
\begin{aligned}
\lambda w_{1}^{\prime}\left(w_{1}^{-1}, w_{2}^{-1}\right) & +w_{2}^{\prime}\left(w_{1}^{-1}, w_{2}^{-1}\right)=\left(w^{\prime} \circ f \circ w^{-1}\right)_{1} \\
& =w_{1}^{\prime}\left(w_{1}^{-1}(\lambda x+y, \lambda y), w_{2}^{-1}(\lambda x+y, \lambda y)\right),
\end{aligned}
$$

$$
\lambda w_{2}^{\prime}\left(w_{1}^{-1}, w_{2}^{-1}\right)=w_{2}^{\prime}\left(w_{2}^{-1}(\lambda x+y, \lambda y), w_{2}^{-1}(\lambda x+y, \lambda y)\right),
$$

i.e., it commutes with the linear transformation $t(x, y)=(\lambda x+y, \lambda y)$. However, by equating coefficients, we find that the only analytic transformation $u$ which commutes with $t$ is a linear transformation of the form $u=(\alpha x+\beta y, \alpha y), \alpha, \beta$, arbitrary. Since $w^{\prime} \circ w^{-1}$ commutes with $t, w^{\prime}=$ $\left(\alpha w w_{1}+\beta w w_{2}, \alpha w w_{2}\right)$.

By repeated application of (6) we find that

$$
\begin{aligned}
& w_{1}\left(f_{1}^{n}, f_{2}^{n}\right)=\lambda^{n} w_{1}(x, y)+n \lambda^{n-1} w_{2}(x, y) \\
& w_{2}\left(f_{1}^{n}, f_{2}^{n}\right)=\lambda^{n} w_{2}(x, y),
\end{aligned}
$$


for positive integral $n$. Since $w$ is invertible, the equations (12) define $f^{n}$ uniquely. Similarly $f^{\alpha}$ defined by (8) is clearly analytic at the origin and solving for it, we have $f^{\alpha}(x, y)=\left(\lambda^{\alpha} x+\alpha \lambda^{\alpha-1} y+\cdots, \lambda^{\alpha} y+\cdots\right)$. These iterates of $f$ clearly commute with $f$ and have the further properties $f^{\alpha} \circ f^{\beta}=f^{\alpha+\beta}, \lim _{\alpha \rightarrow 0} f^{\alpha}=$ identity.

The following theorem completes the discussion in this section.

THEOREM 2.2. (i) There is a 1-1 correspondence between the set of all transformations $\boldsymbol{g}$ analytic at the origin and satisfying

$$
\boldsymbol{g}(0,0)=(0,0), \quad \boldsymbol{g} \circ \boldsymbol{f}=\boldsymbol{f} \circ \boldsymbol{g},
$$

and the set of all linear transformations $\boldsymbol{u}(x, y)=(\alpha x+\beta y, \alpha y)$.

(ii) If $\left(x_{1}, y_{1}\right),\left(x_{2}, y_{2}\right), \neq(0,0)$ are any two distinct points sufficiently close to the origin, there exists a unique analytic transformation $\boldsymbol{g}$ satisfying (13) and such that $\mathrm{g}\left(x_{1}, y_{1}\right)=\left(x_{2}, y_{2}\right)$, iff $w_{2}\left(x_{1}, y_{1}\right) \neq 0$. If $w_{2}\left(x_{1}, y_{1}\right)=0$ such a transformation exists iff $w_{2}\left(x_{2}, y_{2}\right)=0$, in which case there exists a one parameter family of them.

Proof. Clearly every transformation $\boldsymbol{g}$ defined in a neighborhood of the origin by the equation $\boldsymbol{w} \circ \boldsymbol{g}=\boldsymbol{u} \circ \boldsymbol{w}$, has the properties (13). Conversely if $\boldsymbol{g}$ satisfies (13) then $\boldsymbol{w} \circ \boldsymbol{f} \circ \boldsymbol{g}=\boldsymbol{w} \circ \boldsymbol{g} \circ \boldsymbol{f}$ and $\boldsymbol{w} \circ \boldsymbol{g}$ is a solution of (6). Thus by theorem $21 \boldsymbol{w} \circ g$ satisfies $w \circ g=\boldsymbol{u} \circ \boldsymbol{w}$ for some linear transformation $\boldsymbol{u}$ of the class stated.

(ii) If $w_{2}\left(x_{1}, y_{1}\right) \neq 0$, put

$$
\begin{aligned}
& \alpha=w_{2}\left(x_{2}, y_{2}\right)\left[w_{2}\left(x_{1}, y_{1}\right)\right]^{-1} \\
& \beta=\left[w_{1}\left(x_{2}, y_{2}\right) w_{2}\left(x_{1}, y_{1}\right)-w_{2}\left(x_{2} y_{2}\right) w_{1}\left(x_{1}, y_{1}\right)\right]\left[w_{2}\left(x_{1}, y_{1}\right)\right]^{-2}
\end{aligned}
$$

and let $\boldsymbol{u}$ be the linear transformation $\boldsymbol{u}(x, y)=(\alpha x+\beta y, \alpha y)$. Let $\boldsymbol{g}$ be defined by $\boldsymbol{w} \circ \boldsymbol{g}=\boldsymbol{u} \circ \boldsymbol{w} \cdot \boldsymbol{g}$ then has the properties (13) and moreover $w \circ g\left(x_{1}, y_{1}\right)=\boldsymbol{\omega} \circ w\left(x_{1}, y_{1}\right)=w\left(x_{2}, y_{2}\right)$. Since $w$ is invertible locally, we have $g\left(x_{1}, y_{1}\right)=\left(x_{2}, y_{2}\right)$ as required. If $w_{2}\left(x_{1}, y_{1}\right)=0=w_{2}\left(x_{2}, y_{2}\right)$ put $\alpha=\left[w_{1}\left(x_{1}, y_{1}\right)\right]^{-1}\left[w_{1}\left(x_{2}, y_{2}\right)\right]$ in $u$ and let $\beta$ be arbitrary. The equation $\boldsymbol{w} \circ \boldsymbol{g}=\boldsymbol{u} \circ \boldsymbol{w}$ then defines a one parameter family of transformations $\boldsymbol{g}$ with the desired properties. Note: $\alpha$ is finite and non zero since by assumption $\left(x_{1}, y_{1}\right),\left(x_{2}, y_{2}\right), \neq(0,0)$. If $w_{2}\left(x_{1}, y_{1}\right)=0, w_{2}\left(x_{2}, y_{2}\right) \neq 0$, there exist no constants $\alpha, \beta$, such that

$$
w_{1}\left(x_{2}, y_{2}\right)=\alpha w_{1}\left(x_{1}, y_{1}\right)+\beta w_{2}\left(x_{1}, y_{1}\right), w_{2}\left(x_{2}, y_{2}\right)=\alpha w_{2}\left(x_{1}, y_{1}\right) \text {, }
$$

i.e. this requirement is necessary as well as sufficient.

\section{The case $\lambda_{1}^{p}=\lambda_{2}$}

We assume $f$ has been reduced to the form (5), and we seek a solution $w$ of (7). Defining 
we have

$$
\begin{aligned}
& w_{1}^{n}(x, y)=\lambda^{-n p}\left[f_{1}^{n}(x, y)-n a\left(f_{2}^{n-1}(x, y)\right)^{p}\right], \\
& w_{2}^{n}(x, y)=\lambda^{-n} f_{2}^{n}(x, y),
\end{aligned}
$$

Also

$$
\begin{aligned}
& w_{1}^{n}\left(f_{1}, f_{2}\right)=\lambda^{p} w_{1}^{n+1}(x, y)+a\left[w_{2}^{n}(x, y)\right]^{p} . \\
& w_{2}^{n}\left(f_{1}, f_{2}\right)=\lambda^{p} w_{2}^{n+1}(x, y) .
\end{aligned}
$$

$$
\begin{aligned}
& \left|w_{1}^{n+1}-w e_{1}^{n}\right| \leqq|\lambda|^{-n p-p}\left(\left|f^{n+1}-\lambda^{p} f_{1}^{n}-a\left(f_{2}^{n}\right)^{p}\right|+n|a|\left|\left(f_{2}^{n}\right)^{p}-\left(\lambda f_{2}^{n-1}\right)^{p}\right|\right) \\
& \left|w_{2}^{n+1}-w e_{2}^{n}\right| \leqq|\lambda|^{-n-1}\left|f_{2}^{n+1}-\lambda f_{2}^{n}\right| .
\end{aligned}
$$

In this case the estimate of lemma 2.1, must be replaced by two estimates. We note tirst that if $f$ is given by (5) then for some $k>\frac{1}{2}$, we have, $\left|f_{2}(x, y)\right|^{p} \leqq|\lambda y|^{p}+k(|x|+|y|)\left(|x|+|y|^{p}\right)$, for $|x|+|y| \leqq 1$. Choosing $k$ large enough we may also assume that in (5),

$$
\begin{aligned}
& \left|f_{1}(x, y)\right| \leqq\left|\lambda^{p} x\right|+\left|a y^{p}\right|+k(|x|+|y|)\left(|x|+|y|^{p}\right) \\
& \left|f_{2}(x, y)\right| \leqq|\lambda y|+k(|x|+|y|)^{2}, \text { for }|x|+|y| \leqq 1 .
\end{aligned}
$$

Proven in the same manner as lemma 2.1, we now have

Lemma 3.1. Let $s$ be real, $s^{1+1 / p}<|\lambda|<s<1$, and put $\beta=1+|a|$ $(s-|\lambda|)^{-1}$. The region $\Omega=\left\{(x, y) ;|x|+\beta|y|<(s-\lambda)[(1+\beta) k]^{-1}\right\}$, is then contained in $|x|+|y| \leqq 1$, is mapped into itself by $f$, and for all $(x, y) \in \Omega$, and positive integers $n$,

$$
\begin{aligned}
\left|f_{1}^{n}\right|+\beta\left|f_{2}^{n}\right| & \leqq s^{n}(1+\beta) \\
\left|f_{1}^{n}\right|+\beta\left|f_{2}^{n}\right|^{p} & \leqq s^{n v}(1+\beta) .
\end{aligned}
$$

Adapting the methods of theorem 2.1, to the present situation we have

THEOREM 3.1. Let $\left(w_{1}^{n}, w_{2}^{n}\right)$ be defined by (14) and put $w=\left(w_{1}, w_{2}\right)=$ $\lim _{n \rightarrow \infty}\left(w_{1}^{n}, w_{2}^{n}\right)$. Then $w$ exists in a neighborhood of the origin and is an analytic solution of (7). $w_{1}=x+\cdots, w_{2}=y+\cdots$, and any other solution $w^{\prime}$ of (7) with $\boldsymbol{w}^{\prime}(0,0)=(0,0)$ which is analytic at the origin is of the form $w=$ $\left(\alpha^{p} w_{1}+\beta\left(w_{2}\right)^{p}, \alpha w_{2}\right)$ for some constants $\alpha, \beta$.

The transformation $w$ is again invertible in a neighborhood of the origin and so $f^{\alpha}$, defined by (9) for any real or complex $\alpha$, is identically an integral iterate of $f$ if $\alpha$ is a positive integer. The $f^{\alpha}$ commute with $f$ and with each other, and have the same regularity properties as the $f^{\alpha}$ of section 2 . Corresponding to theorem 2.2., we have

THEOREM 3.2. (i) There is a 1-1 correspondence between the set of all transformations $g$, analytic in a neighborhood of the origin and such that

$$
g(0,0)=(0,0), \quad g \circ f=f \circ g,
$$

and the set of all transformations of the form $u(x, y)=\left(\alpha^{p} x+\beta y^{p}, \alpha y\right)$, $\alpha, \beta$ arbitrary constants. (ii) If $\left(x_{1}, y_{1}\right),\left(x_{2}, y_{2}\right), \neq(0,0)$ are any two 
distinct points sufficiently close to the origin, there exists a unique analytic transformation $g$ satisfying (15) and such that $g\left(x_{1}, y_{1}\right)=\left(x_{2}, y_{2}\right)$ iff $w_{2}\left(x_{1}, y_{1}\right)$ $=0$. If $w_{2}\left(x_{1}, y_{1}\right)=0$ such a transformation exists iff $w_{2}\left(x_{2}, y_{2}\right)=0$, in which case there exists a one parameter family of them.

\section{Remarks}

In the case of transformations which are not analytic, some of the above results are valid if terms represented by dots are replaced by terms which are $0\left((|x|+|y|)^{1+\delta}\right), \delta>0$. Even then, those results depending on the existence of $w^{-1}$ are not true in general without further restrictions. For an example of this in the case of one variable see Szekeres [8] page 210.

\section{References}

[1] R. Bellman, The iteration of power series in two variables, Duke Math. J. 19 (1952). $339-347$.

[2] J. Hadamard, Two works on iteration and related questions, Bull. Amer. Math. Soc. 50 (1944), 67-75.

[3] G. Koenigs, Recherches sur les intégrales des certaines équations fonctionnelles, $A n n$. Sci. Ecole Norm. Sup. 3., supp. (1884), 3-41.

[4] L. Leau, Sur les équations fonctionnelles à une ou à plusieurs variables, Ann. Fac. Sci. Toulouse, 11 (1897), E 1-110.

[5] M. Lémeray, Sur le problème de l'itération, C. R. Acad. Sci. Paris 128, (1899), 278.

[6] P. Montel, Lecons sur les recurrences et leurs applications, Gauthier-Villars, Paris, 1957.

[7] A. A. Sarsanov, Analytic iteration of functions of two variables. Soviet Math. 3 (1962), $452-465$.

[8] G. Szekeres, Regular iteration of real and complex functions, Acta Math. 100 (1958), 203-258.

University of California,

Los Angeles. 